Malades psychiques: rendre possible I'accès à bas seuil

Comment des personnes atteintes dans leur santé psychique peuvent-elles bénéficier de soins psychothérapeutiques adéquats? Autrement que partout ailleurs en médecine. Plus de $60 \%$ des patientes et patients consultent un psychiatre de leur propre initiative ou à l'instigation d'une per- sonne autre qu'un médecin. Au vu de la honte des patients et de la stigmatisation des malades psychiques, un accès à bas seuil qui ne crée pas d'obstacle supplémentaire, comme le feraient des franchises différenciées, est indispensable.

Dr méd. Hans Kurt, président SSPP et FMPP

\title{
Papier de prise de position: doublement de la quote-part pour les personnes souffrant de maladies psychiques
}

\section{Gerhard Ebner ${ }^{a}$, Hans Kurt ${ }^{b}$}

a Dr méd., M.H.A., Directeur Universitäre Psychiatrische Kliniken Basel, membre du comité de la Société Suisse de psychiatrie et psychothérapie SSPP

b Dr méd., président de la SSPP et de la Foederatio Medicorum Psychiatricorum et Psychotherapeuticorum FMPP
Correspondances:

Dr Gerhard Ebner M.H.A. Directeur

Universitäre Psychiatrische Kliniken

Wilhelm-Klein-Strasse 27

CH-4025 Bâle

Gerhard.Ebner@upkbs.ch

Dr Hans Kurt

Postgasse 17

CH-Berne 8

kurt@solnet.ch

\section{Charge de santé globale la plus élevée}

Cinquante pour cent de la population souffre dans le cours de sa vie de troubles psychiques, le plus souvent de la dépression, des troubles anxieux, des dépendances ainsi que des douleurs.

Les troubles psychiques représentent la charge globale de santé la plus élevé, impliquant la perte d'un tiers à un quart d'année de vie saine et constituent la principale raison de mise en retraite anticipée. Mille trois cent personnes meurent chaque année en Suisse de suicide, $10 \%$ de la population, c'est-à-dire 750000 personnes en Suisse feront une tentative de suicide dans le cours de leur vie et 90\% d'entre eux souffrent d'un trouble psychique. Uniquement les coûts liés à la conséquence de suicides représentent 2,5 milliards de francs en Suisse et en moyenne quatre proches survivants vivent une souffrance considérable.

\section{Les cliniciens de la médecine} de premier recours sont importants, toutefois en aucun cas suffisants

Dans les faits, un rôle important incombe aux cliniciens de la médecine de premier recours dans la reconnaissance, le traitement et la prévention des troubles psychiques et de leurs conséquences; ceci est confirmé par le fait que près de la moitié des personnes qui se suicident ont fait appel à un médecin de famille dans la semaine précédant le suicide, en règle générale pour une dépression.

Parmi les dépressions par exemple, uniquement la moitié est correctement diagnostiquée, un quart reçoit un soutien médical et seulement $10 \%$ obtiennent un traitement approprié. En ce qui concerne les troubles maniaco-dépressifs, une étude indique clairement que le diagnostic correct n'est posé pour aucun patient dans le cabinet médical. Bien entendu, les médecins de familles disposent aujourd'hui de bonnes connaissances en psychiatrie et sont conscients de la signification des troubles psychiques. Toutefois, tant les études que la pratique médicale indiquent que des soins à large échelle requièrent également des spécialistes, sans lesquels un traitement approprié ne peut pas être administré.

\section{Les personnes concernées veulent aller directement chez le psychiatre}

Les spécialistes de la psychiatrie et les institutions psychiatriques ont une fonction importante à jouer ici, en détectant et en traitant des maladies graves, lesquelles sont généralement curables, en se basant sur leur expérience et sur l'état des connaissances actuelles en matière de traitement. D'après une étude suisse, environ $60 \%$ des malades s'adressent directement à des cabinets de psychiatrie ou à des institutions, sans passer par un médecin de famille. En règle générale, il existe un consensus quant à l'importance de la détection précoce - et du traitement précoce des troubles psychiques pour que l'aide d'un spécialiste médical soit efficace.

La mise en place d'obstacles supplémentaires avec le «Gate-keeping-System», avec l'augmentation de la quote-part pour un groupe de la population - qui d'un point de vue statistique appartient plutôt aux groupes sociaux défavorisés - signifierait une augmentation supplémentaire du seuil d'entrée pour l'accès à des prestations psychiatriques adéquates pour les personnes concernées comme pour leurs proches. Cela aurait un effet très négatif, compte tenu des obstacles déjà existants et de la gêne des malades par rapport à une maladie de l'âme inavouable.

La relation particulièrement sensible entre un médecin et un patient, dans le cas de troubles psychiques devrait garantir un accès direct à cette prestation. De la même manière, une femme ne s'attend pas à devoir se soumettre à un examen préalable par son généraliste en cas de problèmes gynécologiques. Par analogie, il est absurde d'infliger ce détour à des malades psychiques, qui ressentent très souvent une gêne à parler de leur souffrance. A ce propos, un grand nombre 
d'examens indiquent que ce sont justement ces obstacles qui constituent la cause principale de carence de soins des personnes présentant des maladies psychiques.

\section{Collaboration étroite et accès direct au psychiatre}

Que reste-t-il à faire? Une collaboration étroite entre spécialistes de la psychiatrie et généralistes est sans nul doute la clé pour une prévention et un traitement efficace. Ceci est démontré de manière saisissante par certains projets, comme «L'alliance contre la dépression», qui, en plus d'un important travail de relations publiques, s'est également focalisé sur la formation de médecins généralistes et sur la rencontre entre généralistes et spécialistes de la psychiatrie.

\section{Quelques données supplémentaires concernant le papier de prise position précédent}

\section{Prise en charge et épidémiologie}

- Les troubles psychiques sont à l'origine de $1 \%$ des décès.

- Ils représentent la charge globale la plus élevée ( $1 / 4$ à $1 / 3$ des années de vie saines perdues).

- Dans les 20 prochaines années, ils seront en tête de liste de l'OMS en ce qui concerne le DALYs dans les pays à revenus élevés.

- Ils sont la raison principale des retraites anticipées.

- La moitié obtient une aide médicale, $1 / 4$ une aide d'un spécialiste et au total seulement environ $10 \%$ reçoivent une aide médicale adéquate.

- Ils génèrent en Suisse environ $10 \%$ des coûts de traitement.

- Ils occasionnent annuellement en Europe 300 milliards d'euro de coût, dont $1 / 3$ de coût de traitement et $1 / 3$ de coûts indirects.

- 1200-1300 personnes meurent de suicide en Suisse par an.

- $90 \%$ des personnes concernées ont un trouble psychique.

- $10 \%$ de la population fait une tentative de suicide dans le cours de sa vie.

- Plus de $60 \%$ des suicidaires consultent un médecin dans les 4 dernières semaines précédant le suicide, en règle général un généraliste, la plupart du temps avec des symptômes corporels $[1,2]^{*}$.

- Les coûts générés par la conséquence de suicides sont de 2,5 milliards annuellement.

- La stigmatisation semble être l'un des obstacles principaux à la demande de prestations médicales.

$[2,4,5,6,7,8]$

\section{Données liées aux médecins de premier recours}

- Il existe des données empiriques issues de différentes études pour déterminer dans quelle mesure les troubles psychiques sont identifiés par les médecins généralistes. Ces données concernent généralement la détection de troubles dépressifs. Les études allemandes relèvent toutes un taux de reconnaissance de 45 à $50 \%$.

- L'étude actuelle de Sielk [9] dans les cabinets médicaux de Düsseldorf démontre une concordance de près de $50 \%$ entre l'évaluation du médecin et les tests psychiatriques et confirme ainsi les résultats d'études antérieures d'Ebel, Beichert et Berger [10, 11].

- Des taux de détection plus élevés sont rapportés dans une étude de Jacoby et al. [12]. Cette étude rapporte des taux de détection de la dépression majeure situés entre 50 et $75 \%$. Cette étude met toutefois également en évidence le taux de faux positif de $11,3 \%$, c'est-à-dire le taux de patients pour lesquels le diagnostic était erroné, qui ne remplissaient ni les critères DSM ni CIM pour la dépression, mais qui étaient tout de même traités d'un point de vue médicamenteux. Avec 15\%, Berger [11] rapporte un taux de faux positifs encore plus élevé.
- Dans l'étude américaine de Das et al. [13] portant sur des patients bipolaires et sa détection dans les cabinets de généralistes, près de $50 \%$ des patients bipolaires sont diagnostiqués pour dépression et aucun (!) n'a été diagnostiqué avec un trouble bipolaire.

- Ebel et Beichert [10] rapportent que 50-75\% des dépressions restent non traitées, un traitement médicamenteux se fait dans $15 \%$ des cas, toutefois dans la grande majorité des cas, le dosage n'est pas suffisant. Uniquement $10 \%$ des patients dépressifs sont envoyés à un médecin spécialiste, toujours selon les mêmes auteurs. Jacoby et al. [12] rapportent des taux similaires de $10 \%$ à $16 \%$ d'envoi à des spécialistes des patients diagnostiqués avec une dépression.

- Althaus et al. [14] partent du principe, que le taux de patients dépressifs traités adéquatement se situe seulement entre $10 \%$ et $20 \%$.

- Environ $60 \%$ des patients recherchent directement l'aide auprès d'un psychiatre [15]. sous trouverez la

www.bullmed.ch $\rightarrow$ archives $\rightarrow 2009 \rightarrow 49$. 


\section{Références}

1 Walther F, Noack H. Suizid und Suizidversuch in der ärztlichen Praxis. Ergebnisse einer retrospektiven, epidemiologischen-sozial-wissenschaftlichen Untersuchung. Bern: Institut für Sozial- und Präventivmedizin;1988.

2 Michel K. Der Arzt und der suizidale Patient. Schweiz Med Forum. 2002; 29/30: 704-7.

3 Goldsmith SK et al. Barriers to effective Treatment and Intervention. In: Reducing Suicide a national imperative. Washington D.C. 2002; 331ff. www.nap.edu

4 Ajdacic-Gross V, Graf M. Bestandesaufnahme und Daten zur psychiatrischen Epidemiologie in der Schweiz; 2003.

5 Burns $\mathrm{T}$ et al. Which individuals with affective symptoms seek help? Results from the Zurich epidemiological study. Acta Psychiatr Scand. 2003;108:419-26.

6 Obsan. Suizide und Suizidversuche. Indikatoren. 2009; www.obsandaten.ch

7 Schuler D, Rüesch, P. Psychische Gesundheit in der Schweiz. Monitoring. Schweizerisches Gesundheitsobservatorium. Neuchâtel. www.obsan.ch

8 Wittchen HU, Jacobi F. Size and burden of mental disorders in Europe - a critical review and appraisal of 27 studies. European Neuropsychopharmacology: the Journal of the European College of Neuropsychopharmacology. 2005; (4)15:357-76.
9 Sielk M, Altiner A, Janssen B et al. Prävalenz und Diagnostik depressiver Störungen in der Allgemeinarztpraxis. Psychiat Prax. 2009;36:169-74.

10 Ebel H, Beichert K. Depressive Störungen bei Patienten der Allgemeinmedizin. Deutsches Ärzteblatt. 2002;99(3):A124-A130.

11 Berger M. Die Versorgung psychisch Erkrankter in Deutschland - unter besonderer Berücksichtigung des Faches «Psychiatrie und Psychotherapie». Nerven-arzt. 2004.75:195-204

12 Jacoby F, Höfler M, Meister W, Wittchen HU. Prävalenz. Erkennens- und Verschreibungsverhalten bei depressiven Syndromen - Eine bundesdeutsche Hausarztstudie. Nervenarzt. 2002;73:651-58.

13 Das AK, Olfson M, Gamer-off MJ et al. Screening for Bipolar Disorder in a Primary Care Practice. JAMA. 2005;293(8):956-63.

14 Althaus D, Niklewski G, Schmidtke A, Hegerl U. Veränderung der Häufigkeit suizidaler Handlungen nach zwei Jahren. «Bündnis gegen Depression». Nervenarzt. 2007;78:272-82.

15 Boggio Y et al. Evaluation de la mise en œevre et des effets immédiats de la nouvelle ordonnance du DFI sur les prestations dans l'assurance concernant la psychothérapie (OPAS). Evaluanda. Genève: Interface Luzern; 2008 